\title{
A Brief Discussion on Mixed Micelle Formed by Block Copolymer and Surfactant
}

\author{
Narayani Ghosh $^{\mathrm{a}^{*}}$ and Ramakanta Mondal ${ }^{\mathrm{b}}$ \\ ${ }^{a}$ Department of BSH, University of Engineering \& Management (UEM), Kolkata \\ ${ }^{\mathrm{b}}$ Department of Chemistry, KhatraAdibasiMahavidyalaya, Khatra, Bankura, West Bengal \\ *Corresponding Author: narayani.ghosh@uem.edu.in
}

\section{Abstract}

Amphiphilic block copolymers undergo self-aggregates into various nanostructures in aqueous medium and the properties of block copolymers are highlyaltered by the addition of ionic as well as non-ionic surfactants.By mixing with block copolymers with surfactant, the nano-structure as well as the solubility, stability and bioavailability enhances. In this review we want to provide a briefoverview of mixed-micelle formation between various block copolymers and various non-ionic, cationic, anionic surfactants.

\section{Introduction}

The continuous progress of drug delivery systems has been determined by the requirement of clinical pharmacotherapy to improve therapeutic effect and lesser adverse reaction. Amphiphilic block copolymershaving both hydrophilic as well ashydrophobic parts,self-assemble and form micelles. Ithas gained growing attraction over the lastfew decades in the applications of drug delivery due to many reasons [1]. Micelles are surfactant aggregates having various sizes. Although the classical image of a micelle is a sphere, but contrary to expectations, such spherical micelles are rarely encountered. In order to facilitate geometric packing requirements, micelles more commonly assume ellipsoidal, disk- shaped and rod-like structures [2]. They are considered to be colloids having their sizes which normally span between 1-20 nm. Under a given condition of temperature, pressure and solvent (water, and other polar and protic solvents), the individual surfactant monomers start to aggregate, and micelles formation occur at a particular threshold concentration of the surfactant. This specific concentration is usually referred to as the "Critical Micelle Concentration" (CMC)[3-5]. Micelles are believed to have a hydrocarbon core which is dry in nature because of hydrophobic interactions amongthe hydrocarbon tails of the surfactant and the polar/charged hydrophilic head groups exposeto bulk polar solvent due to hydrophilic interactions among the polar or charged head groups and the solvent molecules 
[5].Various techniques such as light scattering [6,7], fluorescence spectroscopy [8,9], NMR, specific volume [10], and small-angle neutron scattering (SANS) [11] have been generally used to understand the aggregation behaviour of these systems.Block copolymeric micelles having a core-shell structure can dissolvepoorly water-soluble drugs into its hydrophobic inner core by hydrophobic interaction and protect the incorporated drugs from inactivation in biological media. Also, hydrophilic blocks can form the outer hydrophilic layer of micelles which can protect loading drugs from outside environment to enhance the stability.Pluronic block copolymers containing hydrophilic poly ethylene oxide (PEO) and hydrophobic poly propylene oxide(PPO) blocks which are arranged in tri-block PEO-PPO-PEOstructures or diblock PEO-PPO structure. Due to low CMC of the block copolymer,it has a strong micellar stabilitytowards dilution, therefore in the micelle-bound drug safely transport through the blood circulation $[12,13]$. Mixed amphiphile systems which includes polymers, copolymers and surfactants have captured significant attention due to the complex ways they aggregate into "supramolecular", "nanoscale" and "self-assembled" structures and their applications in chemical industry [14-17]. Mixed systems consist of polymer-surfactant have unusualcolloidal characteristics, physicochemical properties, detergency, foaming behaviour compared to a single micellar system [18,19]. Numerousmodels have been established to describe the polymer-surfactant interactions by a spectroscopic, electrical andlight scattering techniques[17, 20-24]. It is reported that the addition of surfactantsleads to modify the structure of the polymer micelles. It is mostly reported that the size of mixed micelles form between surfactants and block copolymers gradually reduced with increasing amounts of surfactants [25-27]. However, in some cases, larger mixed micelles are formed [28,29].In this review, we present an overview of studies on mixed micelle formation between block copolymers and surfactants.

\section{Mixed micelle formation between block copolymer and non-ionic surfactant}

Mixed micelles of block copolymer-nonionic surfactant have higher stability and display better biocompatibility and lower toxicity as compare to ionic surfactants [16,30-31]. Qiaoetal. [32] have examined a series of Triton $\mathrm{X}$ surfactants havingvarious ethylene oxide chain length and poly(ethyleneglycols) (PEG) having 
different molecular weight (MW), to see the effects of length of polymer chain on the cloud point of the surfactants. They have found that PEGs with low molecular weight are supposed to form complexes with micelles where the PEG is adsorbed on themicellar surface, as the short chains can bind to themicelle. Glycols having higher molecular weight can form a different type of polymer-surfactant complex where the glycol bound to the surfactant formrandom coil with its hydrophilic headsdirected towater. As a result of these PEGs with low MW increase the cloud point $(\mathrm{CP})$ of Triton $\mathrm{X}$ 100 but those with a higher MWdiminish the CP. Ge et al. [33] investigated the structure of Triton X-100 (Triton X100)/PEG by fluorescence resonance energytransfer (FRET), dynamic light scattering (DLS), freeze-fractured transmission electron microscopy (FFTEM) and proton NMR techniques. The FRET study shows that the addition of PEG results in the increase of distancebetween the donor and acceptor moiety in Triton X-100 micelle, signifying that PEG chains penetrate into Triton $\mathrm{X}$ 100 micelles making thePEG-bound Triton $\mathrm{X}-100$ aggregates looser than that of free Triton X-100 micelles and it is independent of the molecular weight of glycol. PEG forms Triton X-100/PEG complex by the absorption on the surface or incorporation into the outer layer of the micelles which id hydrophilic in nature, for shorter chains of PEG $(\mathrm{MW}<2000)$ it displays a sphere-like structure and for longer chains of PEG $(\mathrm{MW}>2000)$ it shows the coral-shaped clusters. Ge et al. [34]has studied the complex formation between Triton X-100 and triblock copolymer F127 by DLS, isothermal titration calorimetry (ITC) and ${ }^{1} \mathrm{H}$ NMR spectroscopy. They have showed that the mixed aggregates formation of F127 with Triton X-100 depends on the concentration of polymerand temperature. At lower Triton X-100 concentration $<9.42 \mathrm{mM}$, smaller F127/Triton X-100-100 complexes are formed as F127 unimers cover around Triton X-100 micelles with Triton X-100 micelle acts as skeleton at lower temperature. At higher temperature F127 micelle formation occurs which leads to the collapse of Triton X-100 micelles, and the larger F127 micelle- Triton X-100 monomer aggregation is formed. At the intermediate concentration region of Triton X-100 (9.42-94.85 mM), TX-100 micelles promote the formation of F127 micelles and F127/Triton X-100 aggregation is dominated with the skeleton of F127 micelle. However, the skeleton of F127 disrupts due to heating and smaller complexes of Triton X-100 micelles-F127 monomers are formed.The saturation of interaction with F127 and Triton X-100 is 
achieved at higher Triton X-100 concentration region $(>157.57 \mathrm{mM})$.Costa and co-workers [35] have reported the studies of the non-ionic surfactant $\mathrm{C}_{12} \mathrm{EO}_{6}$ and the triblock copolymer $\mathrm{EO}_{20} \mathrm{PO}_{68} \mathrm{EO}_{20}$ (P123) at various temperature range by static and dynamic light scattering. In aqueous medium, micelles with spherical shape are formed by the self-aggregation of P123 with $10 \mathrm{~nm}$ hydrodynamic radius. Addition of $\mathrm{C}_{12} \mathrm{EO}_{6}$ into that selfassembled structure of $\mathrm{P} 123$ resulted mixed micelles formation.The mixed micelle progressively changes its structure from spherical shape to polymer-like with increasing surfactant concentration. At low $\mathrm{C}_{12} \mathrm{EO}_{6} / \mathrm{P} 123$ molar ratios (less than12), the molar mass of the mixed micelles reduces with an increasing concentration of $\mathrm{C}_{12} \mathrm{EO}_{6}$ in the mixed micelles for all examined temperatures. In this region, spherical the mixed micelles form and its hydrodynamic radius decreases. An exception of this observation isnoticed for $\mathrm{C}_{12} \mathrm{EO}_{6} / \mathrm{P} 123$ molar ratios between 2 to 3 , where rode-like structure is found at $40^{\circ} \mathrm{C}$. At molar ratios of 48 and above that, the polymer-like micelles are formed by concentration-induced growth. Kumbhakar et al. [36]has investigated the mixedmicelle aggregationbehaviour of P123 andTriton X-100. They have observed that the incorporation of Triton $\mathrm{X}-100$ to the P123 aggregation leads tothe hydration in the corona region of P123 micelles. Zhu and co-workers [37]have recently studied the self-assembled structure of F127 and a nonionic surfactant (HS15) with various mixing ratios. The study has established that in water F127 molecules could interact with hydrophobic force with HS15 molecules. It is found that at different F127/HS15 ratios different types ofaggregates can form. Roy et al. [38] has studied the supramolecular aggregates formed between P123 and two bile salts:Sodium Deoxycholate and Sodium Taurocholate with different hydrophobicity. By using dynamic light scattering and different other spectroscopic technique they haveunveil that the bile salts penetrate intothe core-corona region of the P123 micelle. At higher concentration of bile salts there (molar ratio of bile salt and P123 around 41:3) new type ofsupramolecular aggregate are formed with two different types of complex structure. Patel et al. [16] have studied the interactions behaviour of Triton X-100 with non-ionic polyethylene glycols (PEG) and polypropylene glycols (PPG) polymers, as well as triblock PEGPPG-PEG and PPG-PEG-PPG. PEG copolymers. From dynamic light scattering, cloud point measurement and small angel neuron scattering measurement they suggested that PPG and PEG-PPGPEG copolymers give distinct effect on 
Triton X-100 micelles. The structure of triblock copolymers remarkably modifiesby Triton X-100 surfactant as compare to PEG and PPG homo-polymers [16]. At ambient temperature, PEG and PPG form complex with Triton X-100 micelles, but at higher temperature PPG solubilizes Triton X-100 polymer and promotes micellar growth. Interestingly, triblock copolymers (which contain both PEG and PPG moieties) form mixed micelle with Triton X-100 and the structure of the mixed-micelle modified with varying temperature.At a higher temperature elongated mixed micelle aggregates are formed by lower molecular weight hydrophobic pluronics. However, at higher temperature pluronics such as L101 and L121 with high molecular weight providelower flexibility for micellar transition. Hence the characteristic properties of polymer showa vital role in the polymer-surfactant interaction. Thesetypes of informationabout the interaction of polymers with non-ionic micelles arehighlycrucial in surfactant-polymer based preparations.

\section{Mixed micelle formation between} block copolymer and ionic surfactant

Recently anionic surfactant boundblock copolymers have been became popular as it can solubilize wide range of drug molecules as well as it is more viscous compared non-ionic polymer mixed micellar system which is ideal forcontrolled drug delivery, also it provides good templates for nanostructured materials preparation [3941].Triblock copolymers interact with ionic surfactants like surfactant sodium dodecyl sulfate (SDS), cetyl trimethyl ammonium bromide (CTAB) etc., in aqueous media and form mixed-micelles [44].Pluoronic copolymer form mixed micelle with anionic surfactant SDS in lower concentration regime i.e., < $\mathrm{CMC}$ [42]. The hydrophobic interior of the PPO block of bock copolymer solubilizes SDS monomers and form a smaller spherical mixed micelle [42]. Prameela etal. reported that by the addition different mixing concentration of SDS and blockcopolymer we can achieve mixed micelles of different morphologies ranging from spherical to ellipsoidal [39]. Mukherjee et al. have studied the binding properties of a model drug in a mixed micelle. For the study, they have used the phenazinium dye, phenosafranin (PSF) as a model drug and monitored the interaction of PSF with F127 and F127-SDS mixed system [43]. Both spectroscopic and calorimetric experiments prove that the drug binding efficiency gets enhanced when SDS is 
added into F127 micelle and this leads to mixed micelleformation between F127 and SDS. The micro-environment of the mixed micelle becomes more hydrophobic due to formation of more compact mixed-micelle formationthat improve physical stability and enhance drug loading capacities [43]. Jadoon et al. has studied the mixed micelle formation between block co-polymer with both cationic CTAB and anionic surfactant SDS [44]. After mixing of ionic and nonionic block copolymer surfactant the hydrodynamic radius grows into uniform distribution, which signifies that almost all surfactant molecules presentin the solution are in the micellar form [44]. Rehman etal. also has studied the complex formation between amphiphilic non-ionic diblock copolymer with anionic surfactant SDS and surfactant hexadecyltrimethylammonium bromide which is cationic in nature [45]. In mixedmicelle the electrostatic repulsion between surfactant molecules diminished and favored the formation ofsmaller polymerbound micelles [45]. Recently, Sahu and co-workers [46] have studied the mixed micelle formation between F127 and a cationic surfactant dodecyltrimethylammonium bromide (DTAB) at different compositions by excited-state proton transfer. It is shown that the nature of mixed micelle formed between F127 and DTAB is markedly different at low and high concentrations of surfactant at low concentration of DTAB, mixed micelle formed that is highly compact and less hydrated. However, as concentration of DTAB is increased, a less compact and more hydrated mixed micelle formed.

\section{Mixed micelle formation between two different block copolymer surfactants}

To increase the stability and drug loading efficacy Ebrahim Attia et al. studied the mixed-micelle from two or more different block copolymers [47]. They found that the hydrophobic interactions among the blocks copolymers with hydrophobic nature show the importance in the mixed micelles formation [47]. Voets et al.have investigated the mixed micelles formed by two diblock copolymers with oppositely charged and they observed that hydrophobic interactions as well as electrostatic interaction between the oppositely charged blocks play major roles for the formation of mixed-micelle [48]. Dabholkar and his coworkers prepared mixed micelles by the mixing ofpoly(ethylene glycol) 2000 -phosphatidyl ethanolamine conjugate and $D-\alpha-$ tocopheryl polyethylene glycol 1000 succinate in equal molar ratio (1:1)and they found that the anti-cancer drug 
paclitaxel which is almost insoluble can be efficiently solubilized and much stable under various physiological conditions like at low $\mathrm{pH}$ values and in the presence of bile acids [49].

\section{Conclusion}

In this present review, our objectis to focus on the mixed-micelle formation between non-ionic amphiphilic blockcopolymer and different nature of another polymer surfactant. Block copolymers contain covalently link with hydrophilic PEO and hydrophobic PPO block, therefore, micellization occurs in aqueous solution. By carefully choosing the surfactant, one can tune stability and shape of micelle without covalently attachment. Naturally, this will definitely affect the applicability of these micelles in industrial and biomedical applications. Block copolymer mixed micelle enhanced the solubility in the core of the micelle therefore for transporting of poorly watersoluble drugs it can use as a good drug career. Also, in mixed-micelle the encapsulated drug protected from the biologicalharsh environment and deliveredto the target sites in a controlled manner.

\section{Reference}

[1] H. Xu, P. Yang, H. Ma, W. Yin, X. Wu, H. Wang, D. Xu, X. Zhang, Drug Deliv, Early Online: 1-9.

[2]R.J Hunter, Foundations of colloid science. Oxford University Press, New York, pp 1-565(1987).

[3]S. P. Moulik, Current Science,71, 368 (1996).

[4]S. S. Berr, J. Phys. Chem.,91,4760 (1987).

[5]T. Telgmann, U. Kaatze, J. Phys. Chem. 104, 1085 (2000).

[6]V. Singh, P. Khullar, P. N, Dave, N. Kaur, Int. J. Ind. Chem., 4, 12 (2013).

[7]T. Thurn, S. Couderc, J. Sidhu, D. M. Bloor, J. Penfold, J. F. Holzwarth, E. Wyn-Jones, Langmuir, 18, 9267 (2002).

[8]S. L. Solan, R. J. Phillips, P. M. Cotts, S. R. Dungan, J Colloid Interface Sci., 191, 291 (1997).

[9]K. Nakashima, T. Anzai, Y. Fujimoto, Langmuir, 10, 658 (1994).

[10]R. K. Williams, M. A. Simard, C. Jolicoeur, J. Phys. Chem., 89, 178 (1985). [11]I. Goldmints, F. K. von Gottberg, K. A. Smith,T. A. Hatton, Langmuir, 13, 3659 (1997).

[12]Z, Jiao, X. Wang, Z. Chen, Asian J. Chemistry, 25, 1765 (2013). 
[13] S. Stolnik, L. Illum, S.S. Davis, Adv.

Drug Deliv. Rev., 16, 195 (1995).

[14] Y. Zheng, H. T. Davis, Langmuir, 16, 6453 (2000).

[15]B. Lindman, G. Karlstrom, Polymer-

Surfactant Systems: The structure, dynamics andequilibrium properties of colloidal systems, NATO ASI Series, Springer Netherlands, (1990).

[16]U. Patel, N. Dharaiya, J. Parikh, V. K. Aswal, P. Bahadur, Colloids Surf. A Physicochem. Eng., 481, 100(2015).

[17] N. V. Sastry, H. Hoffmann, Colloids Surf. A, 250, 247 (2004).

[18]A. Kabalnov, B. Lindman, U. Olsson, L. Piculell, K. Thuresson, H. Wennerstrom., Colloid Polym. Sci. 274, 297(1996).

[19] R. V. Klitzing, Adv. Colloid. Interface Sci., 114, 253 (2005).

[20] K. C. Tam, E. Wyn-Jones, Chem. Soc. Rev., 35,693 (2006).

[21] E. D. Goddard, J. Colloid Interface Sci.256, 228 (2002).

[22] M. S. Bakshi, N. Kaur, R. K. Mahajan, J.Photochem. Photobiol. A, 183, 146 (2006).

[23] E. Pettersson, D. Topgaard, P. Stilbs, O. Soderman, Langmuir 20,1138 (2004).

[24] J. Appell, G. Porte, M. Rawiso, Langmuir 14, 4409 (1998).

[25]J. Jansson, K. Schillé n, G. Olofsson, R. Cardoso da Silva, W. Loh, J. Phys. Chem. B, 108, 82 (2004).
[26]S. Myhre, S. Myhre, L. Willner, K. D. Knudsen, R. Lund, Langmuir, 36,12887 (2020).

[27]J. Jansson, K. Schillen, G. Olofsson, R. Cardoso da Silva, W. Loh, J. Phys. Chem. B, 106, 1239 (2002).

[28]L. M. Bronstein, D. M. Chernyshov, G. I. Timofeeva, L. V. Dubrovina, P. M. Valetsky, A. R. Khokhlov, Langmuir, 15, 6195 (1999).

[29]N. Rehman, A.Khan, I. Bibi, C. I. D. Bica, M. J. Siddiq, Dispers. Sci. Technol., 34, 1202 (2013).

[30] Y. Yang, J. C. Wang, X. Zhang, W. L. Lu, Q. Zhang, J. Control Release, 135,175 (2009).

[31] S. K. Mehta, N. Jindal, Colloids Surf. B, 101, 434 (2013).

[32] L. Qiao, A. J. Easteal, Colloid Polym. Sci., 276, 313 (1998).

[33] L. Ge, X. Zhang, R. Guo, Polymer, 48, 2681 (2007).

[34]L. Ge, R. Guo, X. Zhang, J. Phys. Chem. B 112, 14566 (2008).

[35] K. Schille'n, J. Jansson, D. Lo1f, T. Costa, J. Phys. Chem. B, 112, 5551 (2008).

[36] M. Kumbhakar, S. Dey, P. K. Singh, S. Nath, A. K. Satpati, R. Gangully, V. K. Aswal, H.Pal, J. Phys. Chem. B, 115,1638 (2011).

[37] W. Wu, Z. Zou, S. Yang, Q. Wu, W. Li, Q. Ding, Z. Guan, W. Zhu, Langmuir, 36, 2082 (2020). 
[38]A. Roy, N. Kundu, D. Banik, J.

Kuchlyan, N. Sarkar,Phys. Chem. Chem. Phys., 17, 19977 (2015).

[39]G. K. S. Prameela,B. V. N. Phani Kumar, A. Pan,V. K. Aswal,J. Subramanian, A. B. Mandal, S. P. Moulik, Phys. Chem. Chem. Phys. 17, 30560 (2015).

[40] D.Loef, A.Niemiec, K. Schillen, W. Loh, G. A. Olofsson, J. Phys. Chem. B, 111, 5911 (2007).

[41]R.Wang, Y. Tang, Y.Wang. Langmuir, 30, 1957 (2014).

[42]M. Almgren, J. van Stam, C. Lindblad, P. Li, P. Stilbs,P.Bahadur, J. Phys. Chem., 95, 5677 (1991).

[43]R. Mondal, N. Ghosh, S. Mukherjee,J. Phys. Chem. B, 120, 2968 (2016).

[44]Q. Jadoon, I. Bibi, K. Mehmood, S. Sajjad, M. Nawaz, F. Ali, S. Bibi, W. urRehman, S. Bano, M. Usman,Mater. Res. Express 4, 035307(2017).

[45] N. Rehman,A. Khan, I. Bibi, M. Siddiq, Chin. J. Polym. Sci., 30, 217 (2012).

[46] T. Pal, K. Sahu, J. Phys. Chem. B, 123, 8559 (2019).

[47]A. B. Ebrahim Attia, Z. Y. Ong, J. L. Hedrick, P. P. Lee, P. L. R. Ee, P. T. Hammond, Y.-Y. Yang, Curr. Opin. Colloid Interface Sci., 16, 182, (2011).

[48] I. K. Voets, A. De Keizer, M. A. Cohen Stuart, J. Justynska, H. Schlaad, Macromolecules, 40, 2158 (2007).
[49] R. D. Dabholkar, R. M. Sawant, D. A. Mongayt, P. V. Devarajan,V. P. Torchilin, Int. J. Pharm., 315, 148(2006). 